\title{
Tramadol and
}

\section{hypoglycaemia: comparison with other step 2 analgesic drugs}

Cindy Bourne, ${ }^{1}$ Aurore Gouraud, ${ }^{1}$ Amélie Daveluy, ${ }^{2}$

Aurélie Grandvuillemin, ${ }^{3}$ Pascal Auriche, ${ }^{4}$ Jacques Descotes, ${ }^{1}$

Thierry Vial ${ }^{1} \&$ the French Association of Regional

Pharmacovigilance Centres ${ }^{5}$

${ }^{1}$ Centre de Pharmacovigilance, Lyon, ${ }^{2}$ Centre de Pharmacovigilance, Hôpital Pellegrin, Bordeaux Cedex,

${ }^{3}$ Centre de Pharmacovigilance, Dijon, ${ }^{4}$ Agence Française de Sécurité Sanitaire des Produits de Santé,

Saint-Denis and ${ }^{5}$ Association Française des Centres Régionaux de Pharmacovigilance, Lyon, France
Correspondence

Dr Thierry Vial, Centre de

Pharmacovigilance, 162 avenue

Lacassagne, 69003, Lyon, France.

Tel.: +33472116997

Fax: +3347211 6985

E-mail: thierry.vial@chu-lyon.fr

Keywords

adverse drug reaction, analgesic drugs, dextropropoxyphene, hypoglycaemia, tramadol

\section{Received}

30 July 2012

Accepted

28 August 2012

Accepted Article

Published Online

4 September 2012

\section{WHAT IS ALREADY KNOWN ABOUT} THIS SUBJECT

-Whereas dextropropoxyphene (DXP) has been recognized as a possible cause of hypoglycaemia, only isolated reports have described a similar adverse effect with tramadol (TRM).

- To the best of our knowledge, there is no large study comparing the occurrence of hypoglycaemia in patients treated with these step 2 analgesics.

\section{WHAT THIS STUDY ADDS}

- The aim of this study was to describe spontaneous reports of hypoglycaemia associated with TRM and to compare these data with that of DXP-induced hypoglycaemia.

- The results show that the characteristics of hypoglycaemia in tramadol users are very similar to those described with DXP, with old age, diabetes and renal insufficiency as the main risk factors.

- Physicians and patients should be warned of this potential adverse effect.

\section{AIMS}

The risk of hypoglycaemia with tramadol (TRM) is not well described. Our aim was to analyze spontaneous reports of hypoglycaemia registered in the French Pharmacovigilance database and to compare these data with two other step-2 analgesic drugs.

\section{METHODS}

Cases of hypoglycaemia associated with TRM, dextropropoxyphene (DXP) and codeine (COD) recorded between 1997 and November 2010 in the French pharmacovigilance database were compared.

\section{RESULTS}

Seventy-two cases of hypoglycaemia associated with DXP and 43 with TRM were retained for evaluation (the single case reported with COD was not further considered). Most patients were elderly people with no significant difference in age between DXP- and TRM-treated patients (71.2 \pm 21 vs. $69.4 \pm 22.5$ years). Hypoglycaemia occurred after a median of 4 and 5 days with DXP and TRM treatment, respectively. The mean lowest serum glucose concentration was $2.1 \pm 0.9 \mathrm{mmol}^{-1}$ in the DXP group compared with $2.5 \pm 1 \mathrm{mmol}^{-1}$ in the TRM group $(P=$ 0.072). At least, one risk factor of hypoglycaemia was found in most patients, with no significant difference between groups $(58.3 \%$ in the DXP group and $58.1 \%$ in the TRM group). In particular, $31.9 \%$ patients from the DXP group had diabetes compared with $41.8 \%$ from the TRM group $(P=0.28)$ and $18 \%$ of DXP patients had renal insufficiency compared with $16.3 \%$ of TRM patients $(P=0.8)$.

\section{CONCLUSIONS}

Our study confirms that TRM is associated with the occurrence of hypoglycaemia in elderly or predisposed patients, with characteristics similar to those previously reported with DXP. 


\section{Introduction}

Tramadol (TRM) is a centrally acting weak opioid analgesic, which belongs to the step 2 analgesic classification according to the World Health Organization. It was marketed in France in 1997. TRM is used either alone or in association with paracetamol (acetaminophen) for the treatment of moderate to moderately severe pain. TRM acts through the activation of $\mu$-opioid receptors and in addition inhibits the central re-uptake of serotonin and norepinephrine, which are both involved in central nociceptive transmission [1]. The most frequent adverse effects associated with TRM include gastrointestinal disorders (nausea, vomiting and constipation) and neuropsychiatric effects (sedation, confusion, dizziness, anxiety and sweating) [2].

As recommended by the European Medicines Agency in October 2009, all medicines containing DXP, another weak opioid analgesic, have been withdrawn from the European market in March 2011 because the benefits did not outweigh the risks, in particular in the case of overdoses [3]. Although several investigators have shown that DXP withdrawal from a French hospital was not associated with an increase in step 2 analgesic consumption [4], it is reasonable to anticipate an increase in TRM or codeine (COD) sales in the near future. It is therefore essential to carefully take into account all possible adverse effects associated with these drugs.

To date, only DXP has been clearly associated with the occurrence of hypoglycaemia [5-8]. The first report was published in 1967 [9]. This adverse effect has been mentioned in the summary of the product characteristics (SPC) of DXP for many years and is not mentioned in the SPC of either TRM or COD. Whereas no cases of hypoglycaemia associated with COD have been published so far despite many years of marketing, several isolated cases of TRMinduced hypoglycaemia have been reported in the literature [10-12]. Based on an analysis of spontaneous reports of hypoglycaemia associated with step 2 analgesics and recorded in the French pharmacovigilance database (FPD), the aim of the present study was to investigate whether TRM or COD could be associated with the occurrence of hypoglycaemia, and to compare the characteristics of these hypoglycaemias with those induced by DXP.

\section{Methods}

Spontaneous reports of hypoglycaemia or related terms submitted to the network of the 31 French regional pharmacovigilance centres and recorded in the FPD from 1 January 1997 to 30 November 2010 with drugs containing COD, DXP or TRM coded as suspected or interacting drugs were extracted. Because several cases of true hypoglycaemia were clearly mentioned in the narrative, but mistakenly coded with the clinical symptoms only, two complementary search strategies were performed. The first involved the use of the Medical Dictionary for Regulatory Activities (MedDRA) high level terms containing 'hypoglycaemic conditions' in the coded adverse effect and the second was based on the presence of the strings "\%lyc_mi\%" in the case narrative (\% for truncation and _ to ignore letter with accent).

All cases were carefully reviewed. Cases with insufficient data, unconfirmed hypoglycaemia in the narrative, serum glucose concentrations higher than $3.9 \mathrm{mmoll}^{-1}$, concomitant exposure to any of the selected drugs, obvious other causes such as severe malnutrition, rapid loss of weight, acute alcohol intake, occurrence of hypoglycaemia in a context of suicide attempt, absence of recovery despite drug discontinuation or a Naranjo probability scale indicating a doubtful score [13] were excluded for further analysis. Missing values of glucose concentrations in the narrative were not considered an exclusion criteria provided hypoglycaemia was coded as an adverse effect and suggestive symptoms of hypoglycaemia were associated. Data analyzed for the remaining cases were the patient's characteristics including underlying diseases, the dosage forms, the time to onset of hypoglycaemia after starting the suspected drug, the lowest recorded serum glucose concentration, the presence of clinical symptoms suggestive of hypoglycaemia and the presence of serious criteria defined as any case resulting in death, hospital admission or prolongation of existing hospital stay, persistent or significant disability/incapacity or life threatening. Diabetes and other risk factors known to be associated with hypoglycaemia, such as acute or chronic renal insufficiency, concomitant infection, hepatic insufficiency, alcohol intake, intestinal resection, dysthyroidism, cancer and hyperinsulinism, were also taken into account. Patients were considered to have diabetes if this was clearly mentioned in the narrative, coded in the relevant medical history field or when concomitant exposure included antidiabetic drugs. Regarding the possible presence of clinical symptoms, cases were classified as asymptomatic, minimally or moderately serious (malaise, visual disorders, paraesthesia ...), or serious when neurological signs (coma, convulsions) were mentioned. Variables are compared using the $\chi^{2}$ test and Student's $t$-test with a significance threshold at $5 \%$.

\section{Results}

From 1 January 1997 to 30 November 2010 and whatever the severity criteria, 3025 cases computerized in the FPD involved DXP as a suspected or interacting drug, 3726 involved TRM and 2349 involved COD. Hypoglycaemia was coded as the main symptom or appeared in the narrative in 90 reports for DXP (2.98\% of the total), 53 for TRM $(1.42 \%)$ and two for COD $(0.08 \%)$. As compared with other adverse effects, the reporting rate of hypoglycaemia associated with DXP was higher than that of TRM $(P<0.001)$. Of 


\section{Table 1}

Characteristics of the patients and hypoglycaemia

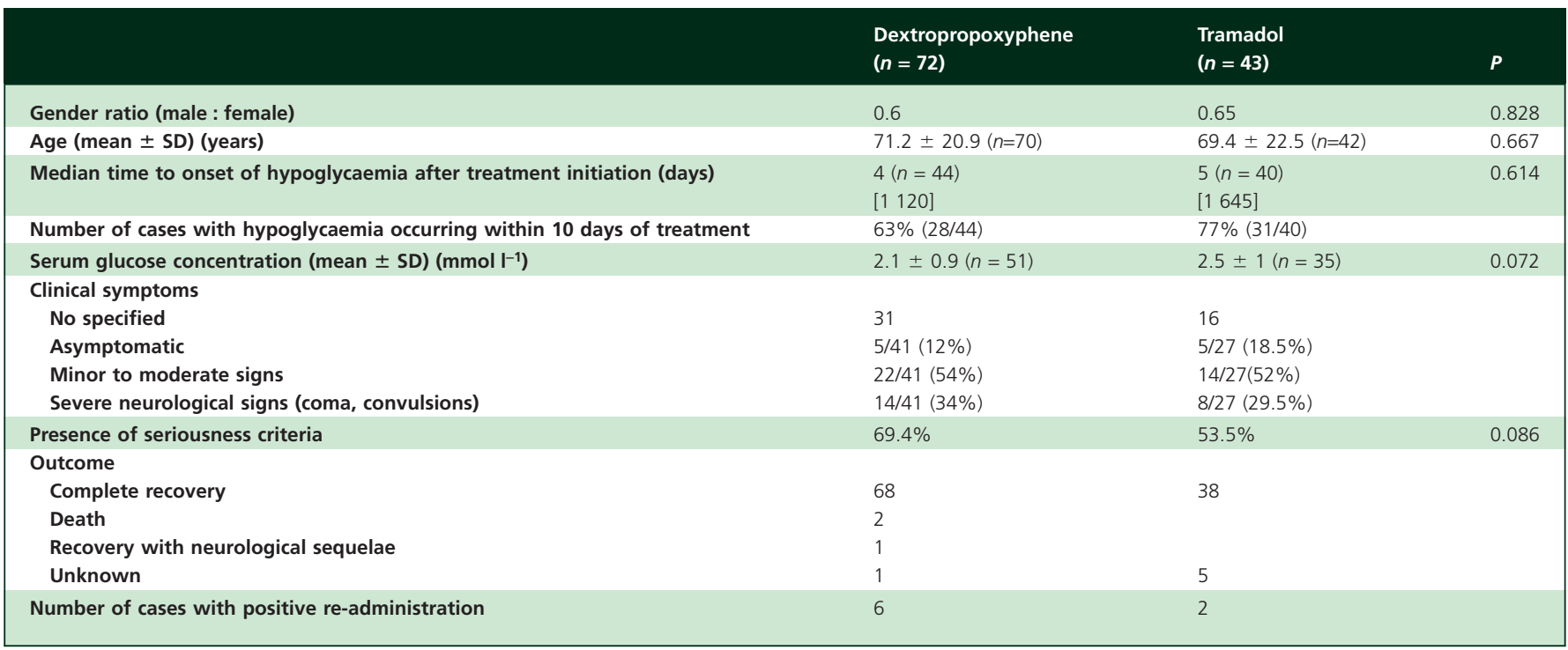

these reports and according to our criteria, 72 and 43 cases of hypoglycaemia associated with DXP and TRM, respectively, were retained for further analysis. As only one case of hypoglycaemia was associated with COD, only DXP and TRM were subsequently compared.

DXP was involved as a single component in three cases, in combination with paracetamol in 59 cases or with paracetamol and caffeine in 10 cases. TRM was involved alone in 36 cases (16 immediate-release forms, 15 sustainedrelease forms, five not stated) and in combination with paracetamol in seven cases. According to the Naranjo probability scale, the mean score in the 43 cases of tramadol associated with hypoglycaemia was 4.2 (range 3 to 8 ).

The main characteristics of the patients and hypoglycaemic events are presented in Table 1. Patients experiencing hypoglycaemia were generally elderly people. There was no significant difference between DXP- and TRMtreated patients $(71.2 \pm 20.9$ years vs. $69.4 \pm 22.5$ years). Hypoglycaemia occurred after a median of 4 and 5 days after the initiation of DXP and TRM treatment, respectively, and most cases were observed within 10 days of treatment. The mean lowest serum glucose concentration was $2.1 \pm 0.9 \mathrm{mmol} \mathrm{I}^{-1}$ in the DXP group $(n=51)$ compared with $2.5 \pm 1 \mathrm{mmol}^{-1}$ in the TRM group $(n=35)(P=0.072)$. When the presence or absence of symptoms were clearly mentioned in the narrative, clinically symptomatic hypoglycaemia was observed with similar frequency with DXP (36 of 41 cases, $87.8 \%$ ) and TRM (22 of 27 cases, $81.5 \%$ ) groups ( $P$ $=0.47)$, whatever the severity of the symptoms. Overall, severity criteria were coded in $69.4 \%$ in the DXP group and $53.5 \%$ in the TRM group $(P=0.086)$. Death occurred in two patients from the DXP group. A 95-year-old patient who experienced glucose-resistant hypoglycaemia with coma died 4 days later from an infectious pneumonitis. Unfortunately, the cause of death was not detailed in another patient aged 86 years, who also experienced severe and glucose-resistant hypoglycaemia $\left(1.1 \mathrm{mmol} \mathrm{I}^{-1}\right.$ at admission). Of note, six patients from the DXP group and two from the TRM group experienced recurring hypoglycaemia after subsequent exposure to the same drug.

As shown in Table 2, at least one predisposing or associated factor was reported in $58.3 \%$ of DXP-treated patients and $58.1 \%$ of TRM-treated patients with no significant difference between groups. Underlying diabetes was the most frequent risk factor reported in $31.9 \%$ of patients in the DXP group and $41.8 \%$ in the TRM group $(P=0.283)$. Among diabetic patients, those from the DXP group were more likely to have non-insulin dependent or insulin requiring diabetes mellitus than those from the TRM group $(P=0.049)$, whereas insulin dependent diabetes was more frequently found in the TRM group $(P=0.019)$. Renal insufficiency was also found in $13(18 \%)$ DXP-treated patients and seven (16.3\%) TRM-treated patients ( $P=$ 0.808). After taking into account the presence of renal insufficiency and several known triggering factors of hypoglycaemia such as cancer, moderately reduced nutrition, sepsis, alcohol consumption, liver disease and thyroid disorders, concomitant risk factors were identified in 28 (38.9\%) DXP users and in 15 (34.8\%) TRM users ( $P=0.668)$.

\section{Discussion}

This study suggests an association between TRM, either alone or combined with paracetamol and the occurrence of hypoglycaemia, without overt difference compared 
Table 2

Predisposing factors

\begin{tabular}{|c|c|c|c|}
\hline & $\begin{array}{l}\text { Dextropropoxyphene } \\
(n=72)\end{array}$ & $\begin{array}{l}\text { Tramadol } \\
(n=43)\end{array}$ & $\boldsymbol{P}$ \\
\hline Diabetes & $23(31.9 \%)$ & $18(41.8 \%)$ & 0.283 \\
\hline Insulin-dependent diabetes & 4 & 10 & 0.019 \\
\hline Insulin-requiring diabetes & 1 & 2 & 0.049 \\
\hline $\begin{array}{l}\text { Non-insulin dependent } \\
\text { diabetes }\end{array}$ & 15 & 5 & 0.049 \\
\hline Not otherwise stated & 3 & 1 & \\
\hline $\begin{array}{l}\text { Other risks factors except } \\
\text { diabetes (and/or) }\end{array}$ & $28(38.9 \%)$ & $15(34.8 \%)$ & 0.668 \\
\hline $\begin{array}{l}\text { Acute or severe chronic } \\
\text { renal failure (creatine } \\
\text { clearance }<30 \mathrm{ml} \mathrm{min}^{-1} \text { ) }\end{array}$ & 6 & 5 & \\
\hline $\begin{array}{l}\text { Renal failure without } \\
\text { precision }\end{array}$ & 7 & 2 & \\
\hline Concomitant infection & 2 & 5 & \\
\hline Hepatic failure & & 1 & \\
\hline Alcohol & 3 & 2 & \\
\hline Enteric resection/anorexia & 2 & 1 & \\
\hline Dysthyroidism & 1 & 1 & \\
\hline Cancer & 6 & & \\
\hline Hyperinsulinism & 1 & & \\
\hline $\begin{array}{l}\text { Both diabetes and other risk } \\
\text { factors }\end{array}$ & $58.3 \%$ & $58.1 \%$ & 0.936 \\
\hline
\end{tabular}

with DXP. These results are in accordance with those of Tavassoli et al. who found a similar reporting rate of hypoglycaemia with DXP (0.8/100 000 person-years) and TRM (0.6/100 000 person-years) [2]. However, this study provided no details on the characteristics of hypoglycaemia. Five isolated case reports also detailed the occurrence of hypoglycaemia in TRM-treated patients, among whom three were over 80 years of age and two were young insulin-dependent diabetes mellitus patients [10-12]. In three of these cases, hypoglycaemia was noticed between 1 to 3 days of treatment and three patients had severe clinical symptoms. Very similar findings were evidenced in our study with hypoglycaemia being usually observed within the first 10 days of TRM treatment, particularly in elderly and predisposed patients, and marked by the presence of mild to severe symptoms in approximately half of the patients. All these characteristics were very close to those observed with DXP, another weak opioid analgesic well-known to be associated with hypoglycaemia [5-9], whereas no convincing cases have been reported with COD. A predisposing factor of hypoglycaemia was found in more than half of the cases, with age, renal insufficiency and diabetes mellitus being the most frequent factors. This suggests that prescribing TRM in such patients should be cautious. The number of patients with diabetes ranged from 32 to $42 \%$ in this study, a rate that appeared rather low given the fact that true hypoglycaemia without diabetes is rare. Unfortunately, and given the limitations of spontaneous notifications, it was not possible to confirm or not the presence of diabetes in other cases. Although noninsulin-dependent diabetes mellitus was more frequently reported among diabetic patients from the DXP group compared with the TRM group, this finding was based on a small number of patients and could have been coincidental or resulting from multiple comparisons. Although a drug interaction might explain this finding, to the best of our knowledge, there are no data suggesting a possible pharmacokinetic interaction between DXP and oral hypoglycaemic drugs.

The mechanisms of TRM-induced hypoglycaemia are still hypothetical and at least two possible explanations based on TRM pharmacological effects can be discussed. A putative serotoninergic-mediated mechanism is substantiated by experimental data showing that serotonin can increase insulin concentration in mice [14]. In rat models of diabetes, serotonin also induced $\beta$ endorphin release and stimulated muscle glucose utilization through the activation of $\mu$-opioid receptors by a mechanism, which is neither dose nor insulin dependent [15]. In addition, hypoglycaemia associated with selective serotonin re-uptake inhibitor treatment has also been reported, but the clinical evidence is limited to few case reports $[16,17]$.

Hypoglycaemia resulting from the activation of opioid $\mu$-receptors by TRM is a more attractive hypothesis. Experimental data in streptozotocin-induced diabetic rats evidenced a dose-dependent lowering of plasma glucose concentrations shortly after intravenous TRM administration at doses ranging from 15 to $150 \mu \mathrm{g} \mathrm{kg}^{-1}$ [18]. This hypoglycaemic effect of TRM was blocked by pretreatment with opioid $\mu$-receptor antagonists such as naloxone. By contrast, only a moderate effect on plasma glucose concentrations was found in normal rats receiving a much higher TRM dose (i.e. 1 to $5 \mathrm{mg} \mathrm{kg}^{-1}$ ). The same authors had previously shown that opioid $\mu$-receptors stimulation lowered plasma glucose in diabetic rats and that loperamide, another $\mu$-receptor agonist, exerted the same effect as TRM [19]. Further investigations indicated that activation of these receptors could decrease plasma glucose concentrations by increasing glucose utilization in peripheral tissues, probably via an insulin-independent mechanism and/or by inhibiting hepatic gluconeogenesis [20]. Although no specific studies have been conducted with DXP, a similar mechanism can probably explain the rare reports of hypoglycaemia in these patients. Overall, these experimental data in diabetic rats are in accordance with our results suggesting that diabetes mellitus appeared as the main risk factor in DXP- or TRM-treated patients who experienced hypoglycaemic episodes.

In conclusion, despite the limitations of our study due to probable under-reporting and the lack of knowledge of the characteristics of the whole treated population, our data indicate that TRM can be associated with hypoglycaemia, particularly in elderly or diabetic patients, or in those with renal insufficiency, similar to what was previously described with DXP. Although further signal strengthening 
studies need to be carried out for definite confirmation, physicians and patients should be warned of this potential adverse effect, which is not mentioned in the summary of the product characteristics.

\section{Competing Interests}

There are no competing interests to declare.

\section{REFERENCES}

1 Raffa RB, Friderichs E, Reimann W, Shank RP, Codd EE, Vaught JL. Opioid and nonopioid components independently contribute to the mechanism of action of tramadol, an 'atypical' opioid analgesic. J Pharmacol Exp Ther 1992; 260: 275-85.

2 Tavassoli N, Lapeyre-Mestre M, Sommet A, Montastruc JL. Reporting rate of adverse drug reactions to the French pharmacovigilance system with three step 2 analgesic drugs: dextropropoxyphene, tramadol and codeine (in combination with paracetamol). Br J Clin Pharmacol 2009; 68: 422-6.

3 European Medicines Agency. Londres: Press release: European Medicines Agency recommends withdrawal of dextropropoxyphene-containing medicines [online]. [updated 2009 Jun 25]. Available at http://www.ema. europa.eu/docs/en_GB/document_library/Press_release/ 2009/11/WC500010365.pdf (last accessed 9 January 2012).

4 Gaubert S, Vié M, Damase-Michel C, Pathak A, Montastruc JL. Dextropropoxyphene withdrawal from a French university hospital: impact on analgesic drug consumption. Fundam Clin Pharmacol 2009; 23: 247-52.

5 Laurent M, Gallinari C, Bonnin M, Soubrie C. Hypoglycemia induced by dextropropoxyphene in very old subjects. 7 cases. Presse Med 1991; 20: 1628.

6 Florez LR, Rozanski J, Castro A, Mintz DH. Propoxyphene-induced hypoglycemia. J Fla Med Assoc 1977; 64: 163-4.

7 Almirall J, Montoliu J, Torras A, Revert L. Propoxyphene-induced hypoglycemia in a patient with chronic renal failure. Nephron 1989; 53: 273-5.
8 Shah P, Aniszweski J, Service FJ. Propoxyphene-induced hypoglycemia in renal failure. Endocr Pract 2006; 12: 170-3.

9 Wiederholt IC, Genco M, Foley JM. Recurrent episodes of hypoglycemia induced by propoxyphene. Neurology 1967; 17: 703-6.

10 Grandvuillemin A, Jolimoy G, Authier F, Dautriche A, Duhoux F, Sgro C. Hypoglycémie lors d'un traitement par tramadol. Presse Med 2006; 35: 1842-4.

11 Taugourdeau S, Chiche L, Rouby F, Default A, Boyer M, Castellan D, Lanfranchi MA, Bornet C, Jean R, Harlé JR, Durand JM, Jean-Pastor MJ. Severe hypoglycemia induced by tramadol: two new cases of an unlisted side effect. Rev Med Interne 2011; 32: 703-5.

12 Jonville-Bera AP, Marie A, Magba D, Gedon E, Autret-Leca E. Tramadol-induced hypoglycemia in a diabetic patient. Therapie 2010; 65: 499-500.

13 Naranjo CA, Busto U, Sellers EM, Sandor P, Ruiz I, Roberts EA, Janecek E, Domecq C, Greenblatt DJ. A method for estimating the probability of adverse drug reactions. Clin Pharmacol Ther 1981; 30: 239-45.

14 Yamada J, Sugimoto Y, Kimura I, Takeuchi N, Horisaka K. Serotonin-induced hypoglycemia and increased serum insulin levels in mice. Life Sci 1989; 45: 1931-6.

15 Chi TC, Ho YJ, Chen WP, Chi TL, Lee SS, Cheng JT, Su MJ. Serotonin enhances beta-endorphin secretion to lower plasma glucose in streptozotocin-induced diabetic rats. Life Sci 2007; 80: 1832-8.

16 Pollak PT, Mukherjee SD, Fraser AD. Sertraline-induced hypoglycemia. Ann Pharmacother 2001; 35: 1371-4.

17 Fernández López MI, Sánchez Esteban J, Jímenez Belló J, Meseguer Zaragoza A. Suggestive episodes of hypoglycemia related to fluoxetine. Aten Primaria 1996; 18: 581.

18 Cheng JT, Liu IM, Chi TC, Tzeng TF, Lu FH, Chang CJ. Plasma glucose-lowering effect of tramadol in streptozotocin-induced diabetic rats. Diabetes 2001; 50: 2815-21.

19 Tzeng TF, Liu IM, Lai TY, Tsai CC, Chang WC, Cheng JT. Loperamide increases glucose utilization in streptozotocin-induced diabetic rats. Clin Exp Pharmacol Physiol 2003; 30: 734-8.

20 Liu IM, Niu CS, Chi TC, Kuo DH, Cheng JT. Investigations of the mechanism of the reduction of plasma glucose by cold-stress in streptozotocin-induced diabetic rats. Neuroscience 1999; 92: 1137-42. 